\title{
Weigh More, Pay More? Public Opinion on Varying Health Insurance Contributions among Divergent Weight Groups
}

\author{
Marie Bernard ${ }^{a, b}$ Steffi G. Riedel-Heller ${ }^{c} \quad$ Claudia Luck-Sikorski ${ }^{a, b}$ \\ a Integrated Research and Treatment Center (IFB) Adiposity Diseases, University Hospital, \\ Leipzig, Germany; ${ }^{\text {b} S R H ~ U n i v e r s i t y ~ o f ~ A p p l i e d ~ H e a l t h ~ S c i e n c e s, ~ G e r a, ~ G e r m a n y ; ~}{ }^{\text {c Institute }}$ \\ of Social Medicine, Occupational Health and Public Health (ISAP), University of Leipzig, \\ Leipzig, Germany
}

Keywords
Obesity $\cdot$ Health insurance $\cdot$ Contributions $\cdot$ Public opinion

\section{Abstract}

Background: The prevalence of obesity and its related costs has increased over the past decades. In Germany, obesity-related costs are merely covered by statuary health insurance. Within the statutory health care system, the health insurance contributions do not differ between people with and without health issues, such as being obese. This study aims to investigate the public's opinion about whether people with obesity should pay a higher proportional health care contribution than people of normal weight. Methods: We conducted a pilot study and collected thereof data of a convenience sample. In total, 179 participants who perceived themselves to be of normal weight $(51.40 \%$ female; mean age $=32.46$, SD $=5.74)$ were surveyed using a questionnaire. Within this questionnaire, the participants had to rate how high the proportional health care contribution for people with and without obesity should be. Moreover, we assessed participants' antifat attitudes by applying the Fat Phobia Scale and the Implicit Association Test. Results: A paired $t$ test revealed that participants suggest a significantly higher proportional contribution for health insurance for people with obesity compared to people with normal weight $(t(178)=4.51, p<0.001)$. Logistic regression analysis indicates that people with stronger explicit $(O R=8.77, p<0.001)$ and implicit stigma $(O R=1.06$, $p=0.018)$, and higher $\mathrm{BMI}(\mathrm{OR}=1.27, p=0.04)$ are more likely to suggest an increased contribution rate for people with obesity. Conclusion: Although we found that participants suggested higher contribution rates for people with obesity, overall only one-quarter of the par- 
Bernard et al.: Weigh More, Pay More?

ticipants suggested higher contribution rates for people with obesity, whereas almost three-quarters of the participants did not distinguish the contribution rate for people with and without obesity. Moreover, we found that the participants called for higher insurance premiums for people with and without obesity. Therefore, future studies should consider giving more information about the statutory health care system or the health care contribution rate before asking participants about their opinion.

(C) 2019 The Author(s)

Published by S. Karger AG, Basel

\section{Introduction}

Obesity has increased substantially over the past decades, and with it, obesity-related health care costs [1]. Since there are different ways to calculate obesity-related costs, there are several estimations of these. In general, distinction can be made between direct and indirect obesity-related costs. Direct costs include not only diagnostic and therapeutic methods, preventive interventions, and rehabilitation therapy, but also the costs of treating the comorbidities of obesity. Depending on how direct costs are calculated, in Germany, they are estimated to be between EUR 863 million and EUR 29.39 billion.

Furthermore, annual indirect costs between EUR 1.4 and EUR 33.65 billion must be added $[1,2]$. These indirect costs refer to the loss of earnings, early retirement, premature death, or limited productivity at work caused by obesity or its comorbidities. When considering a lifetime perspective, Effertz et al. [1] determined an additional burden for the social security system in Germany of EUR 166,911 for every man with obesity and EUR 206,526 for every woman with obesity.

The central social security system in Germany is the statutory health insurance that provides services in the case of illness [3]. It includes most employees, pensioners, their family members, and serves almost $88 \%$ of the population. The remaining $12 \%$ of the German population is privately insured. In this study, we concentrate solely on the statutory health insurance system. The contribution rates of insurance companies amount to $14.6 \%$ of employees' salary. This contribution rate is split equally between employees and employers and is subtracted from the employee's salary [4]. The German statutory health insurance can thus be characterized as a solidary system. The concept of solidarity is also reflected in a risk adjustment scheme that prohibits any additional contribution payments for members of risk groups [5]. Therefore, the proportional contribution is equal for everyone despite individual health risks, such as employees' age or weight status. In other words, a healthy person pays the same proportional contribution as a person with health issues. Moreover, people with a higher income and, therefore, with a higher absolute amount of health care contribution do not receive better treatment than people with a lower contribution. Since a risk adjustment scheme covers for several diseases, such as obesity, the statutory health insurance funds are not disadvantaged by taking in individuals of risk groups. Health insurance funds are thus not tempted to recruit only young and healthy members because of the solidary compensation they will receive for members of specific risk groups [5, 6]. All in all, the German statutory health insurance can be characterized as a solidary system that aims to create a social balance between the more affluent and the poor, the old and the young, and the healthy and the sick [3].

Being obese, however, comes along with stigmatization that might lead to structural discrimination in the form of unfair treatment in several areas of life, such as in the health care market $[7,8]$. Since obesity is often mislabeled as a self-inflicted condition and thus associated with several negative attributes, such as a lack of willpower or being lazy [8], people might suggest a higher contribution rate for those concerned to cover the increased obesity- 
related health care costs. Woolford et al. [9], for example, investigated the public's view on obesity treatment and found increased support to cover the costs of obesity treatment by private insurance. In contrast, they found less support to cover the cost of obesity treatment by public health insurance, particularly for bariatric surgery. Woolford et al. [9] consider the public opinion toward insurance coverage as a potential guideline for public health insurance funds. According to the public's view, insurance funds might adjust the contribution rates to the detriment of people living with obesity. For Germany, there are no data available that investigated this connection in detail. Therefore, this pilot study was conducted to examine the public's opinion on whether and to what extent the contribution rate for the statutory health insurance should be adjusted for weight status.

We assessed implicit and explicit antifat attitudes to measure whether and to what extent participants stigmatize people with obesity. As outlines above, we assume that people who express more explicit or implicit antifat attitudes might claim a higher contribution rate for people with obesity to cover their increased and (perceived) self-inflicted health care expenditure.

\section{Methods}

In this study, we aimed to investigate the public's opinion on whether people with obesity should pay a higher proportion to cover their health insurance. Since this topic has not been investigated before, we conducted a pilot study to get a first insight into the field.

\section{Sample and Recruitment}

We announced the study in public places and online platforms and recruited in this way participants of a convenience sample. This study aimed initially to assess men and women aged between 25 and 45 years of normal weight, which was defined as body mass index (BMI) between 18.5 and $<25.0 \mathrm{~kg} / \mathrm{m}^{2}$. People who stated to meet the inclusion criteria were asked to contact the study team via telephone or email. We then arranged single appointments with each participant and invited them to the Institute of Social Medicine, Occupational Health and Public Health (Leipzig University). Each participant received EUR 20 as payout for participation. Data were collected between October 2015 and October 2016.

\section{Study Design}

Every single appointment was accompanied, conducted, and monitored by a member of the study team. At the beginning of each appointment, the member of the study team gave the participant an overview of the procedure. The participants were then asked to provide their written consent. In the next step, participants were placed in a room just by themselves to fill out a questionnaire in a private atmosphere. Within the survey, participants were initially asked to provide some sociodemographic details. Participants' attitudes toward health care contributions were introduced by a short text stating that the statutory health insurance in Germany is financed by federal subsidy and social security contributions. These contributions are respectively calculated based on employees' income. After this introduction, we implemented questions regarding their attitudes toward health care contributions. After that, participants were asked to complete the Fat Phobia Scale (FPS). After participants completed the questionnaire, they were asked to inform the member of the study team, who waited outside the room. The member of the study team then explained the Implicit Association Test (IAT) to the participants. After participants completed a practice IAT trial and thus showed that they understood the test, they completed the actual IAT trials. Finally, the participants' body weight and height were measured by the member of the study team. 
Bernard et al.: Weigh More, Pay More?

\section{Instruments}

The participants were asked to fill out a digital questionnaire on-site, which included among other things sociodemographic characteristics, implicit and explicit stigma toward people with obesity, and participants' opinion on insurance contributions for different weight groups. All instruments used are described in the following.

\section{Health Care Contributions}

After a short introduction of the topic, participants were asked what proportion of income should be paid by people with and without obesity to cover their health insurance. Therefore, we implemented the following questions: "How high should be the proportional contribution of the statutory health insurance for people with obesity/people of normal weight?" The participants had to input the proportional contribution that should be paid by people with and without obesity, respectively, and could include up to two decimals places. For the analyses, we calculated a variable by subtracting a suggested proportional health insurance contribution for people with obesity from the suggested proportion for people with normal weight. Positive differences indicate that people with obesity should pay a higher percentage, whereby negative differences display the contrary. Zero differences indicate no preferences for a weight group over another. For multivariate logistic analysis, we generated a dichotomous variable, with the value 0 indicating that participants suggested equal contribution rates for people of different weight status, and the value 1 showing higher contribution rates for people with obesity.

\section{Attitudes toward People with Obesity}

To assess implicit attitudes toward weight groups, the IAT was applied. The IAT developed by Greenwald et al. [10] measures automatic associations and thus unconscious prejudices toward specific groups, such as people with obesity. Therefore, participants are asked to match synonyms describing weight status (normal weight vs. obesity) to positive or negative adjectives, such as "good," "bad," "motivated," or "unmotivated" under time constraints. Participants are thereby forced to answer impulsively and reveal their preferences of categorical pairing over another. It is assumed that people with higher implicit stigma toward people with obesity will perform better when they have to match positive adjectives with being thin and negative adjectives with obesity (congruent task) than conversely. In this study, the paper/pencil version of the IAT was applied. After the member of the study team introduced the IAT, the participants completed a short exercise trial (matching positive and negative adjectives with flowers and insects). Afterwards, the participants completed the IAT on attitudes toward weight groups while the member of the study team stopped the time.

The IAT scores were calculated by subtracting the number of correctly classified words in the incongruent task from correctly classified words in the congruent tasks (IAT congruent IAT $_{\text {incongruent }}$ ). Positive differences indicate stronger negative implicit attitudes toward people with obesity, whereas negative differences display a more positive view. The IAT showed excellent reliability for the congruent task (Cronbach's $\alpha=0.95$ ) as well as for the incongruent task (Cronbach's $\alpha=0.93$ ).

Explicit attitudes toward people with obesity were assessed by using the FPS developed by Bacon et al. [11]. The FPS measures explicit stigma by semantic differentials and contains fourteen contrary adjective pairs, such as "lazy" / industrious" or "no will power"/"has will power." Participants are asked to rate a fictive person with obesity on a 5-point scale between these antonyms $(1=$ positive attitudes; $5=$ negative attitudes). Higher FPS scores display stronger explicit stigma toward people with obesity. In this study, the FPS showed good reliability (Cronbach's $\alpha=0.84$ ). 


\section{Sociodemographic Characteristics}

Sociodemographic characteristics, such as educational attainment (highest educational degree) and level of income (monthly household income), were assessed with the questionnaire. A member of the study team measured participants' body weight and height by using an electronic digital scale, accurate to $100 \mathrm{~g}$. A locally manufactured standard measuring board, with increments in millimeters, was used to measure participants' height. The BMI of each participant was calculated for statistical analyses. Although this study aimed initially to assess participants of normal weight, 11 participants were slightly overweight, with a BMI between 25 and 26.3. However, we decided to keep these participants in the sample, since it might give a better insight into the research field.

\section{Statistical Analyses}

All analyses were run with STATA 14.1 [12]. We conducted $t$ tests to analyze whether participants suggested a higher proportional contribution for people with obesity compared to people of normal weight. Further, hierarchy multivariate regression models were calculated to investigate which potential determinants, such as implicit and explicit antifat attitudes, might be associated with the opinion on how high the contribution rates for people with and without obesity should be. Instead of linear regression analysis, we decided on a logistic regression model with the generated dichotomous variable assessing the participants' opinion on whether people with obesity should pay a higher or equal contribution rate compared to people of normal weight as the outcome variable. We did so because the assumption of homoscedasticity was not fulfilled, although we attempted to transform the dependent variable as suggested in the literature [13].

\section{Results}

\section{Sample Characteristics}

Overall, we assessed data of 182 participants. However, we excluded 2 participants who did not speak German fluently and had therefore problems in completing the IAT, and 1 participant who refused to complete the IAT for personal reasons. A total of 179 participants $(51.40 \%$ female; mean age $=32.46, \mathrm{SD}=5.74)$ remained in the study. Sociodemographic characteristics of the sample are displayed in Table 1.

When looking at the antifat attitudes of the examined sample, the following can be stated: 9 participants (5.36\%) showed more positive implicit attitudes toward people with obesity in comparison to people of normal weight, whereas only 1 participant did not show different implicit attitudes toward divergent weight groups. However, the majority of participants (i.e., $94.05 \%(n=158))$ showed more negative implicit attitudes toward people with obesity. A paired $t$ test revealed significant differences for correct attributions made for the incongruent vs. congruent task $(t(167)=19.49, p<0.001)$. In consequence, participants had significantly lower scores when they were asked to attribute positive adjectives (good, motivated, etc.) with obesity and indicate subsequently negative implicit antifat attitudes.

Explicit antifat attitudes, measured by FPS on a 5 -point scale $(1=$ positive attitudes; $5=$ negative attitudes) revealed that the examined sample tended to associate more negative attitudes toward people with obesity ( mean $=3.55, \mathrm{SD}=0.47$ ).

\section{Suggested Health Care Contribution for People with and without Obesity}

The one-sample $t$ tests conducted revealed that participants suggested significantly higher health insurance contribution rates compared to the current average contribution rate 
Bernard et al.: Weigh More, Pay More?

Table 1. Sociodemographic characteristics of participants $(n=179)$

\begin{tabular}{lc} 
Education & \\
No school qualification & - \\
Still in school & - \\
General school (9 years) & $40(22.4)$ \\
Secondary school (10 years) & $8(4.5)$ \\
Technical college (12 years) & $130(62.7)$ \\
Higher school degree ( $\geq 12$ years) & $1(0.6)$ \\
Other & \\
\hline Monthly household income (EUR) & $16(8.9)$ \\
$<500$ & $49(27.4)$ \\
$500 \leq 1,000$ & $43(24.0)$ \\
$1,000 \leq 1,500$ & $24(13.4)$ \\
$1,500 \leq 2,000$ & $11(6.2)$ \\
$2,000 \leq 2,500$ & $9(5.0)$ \\
$2,500 \leq 3,000$ & $8(4.5)$ \\
$3,000 \leq 3,500$ & $2(1.1)$ \\
$3,500 \leq 4,000$ & $6(3.4)$ \\
$4,000 \leq 4,500$ & $6(3.4)$ \\
$>4,500$ (I don't know" & $3(1.7)$ \\
"Prefer not to say" & $2(1.1)$ \\
\hline BMI & $22.16 \pm 1.76$ \\
Age, years & $32.46 \pm 5.74$ \\
\hline Suggested HIC & $18.58 \pm 16.60$ \\
For people with obesity & $16.43 \pm 13.53$ \\
For people of normal weight & $13.94 \pm 9.12$ \\
\hline IAT & $3.58 \pm 0.49$ \\
FPS & \\
\hline \multicolumn{2}{l}{ Values are presented as $n$ (\%) or mean \pm SD. BMI, body mass index; } \\
HIC, health insurance contributions; IAT, Implicit Association Test (IAT \\
difference scores are displayed); FPS, Fat Phobia Scale (1= positive atti- \\
tudes; 5 = negative attitudes). \\
\hline
\end{tabular}

of $14.6 \%$ for people with obesity $(18.6 \% ; t(178)=3.21, p<0.001)$, but also for people of normal weight $(16.4 \%$; $t(178)=1.80, p<0.05)$.

Overall, not a single participant suggested a lower contribution rate for people with obesity compared to people of normal weight. However, we conducted a paired $t$ test to investigate whether the suggested proportional contribution rates for people with and without obesity differed significantly. The $t$ test performed revealed that participants suggested a significantly higher proportional contribution rate for people with obesity compared to people of normal weight $(t(178)=4.51, p<0.001)$. However, only $26 \%$ of participants demanded an increased contribution rate for people with obesity. The remaining $74 \%$ of participants suggested an equal contribution rate for people with and without obesity.

Moreover, we conducted a multivariate logistic regression analysis (Table 2). As explaining variables, we included significantly correlated items, i.e., explicit antifat attitudes (FPS; $r=0.29, p<0.001)$ ), participants' BMI $(r=0.23, p<0.01)$, and household income $(r=$ $-0.26, p<0.001)$. We also included participants' gender, highest educational degree, and age as well as implicit antifat attitudes (IAT) as control variables. Our findings indicate that people with stronger explicit (OR $=8.77, p<0.001)$ and implicit stigma (OR $=1.06, p=0.018)$, and higher $\mathrm{BMI}(\mathrm{OR}=1.27, p=0.04)$ are more likely to suggest an increased contribution rate for people with obesity. 
Bernard et al.: Weigh More, Pay More?

Table 2. Logistic regression model

\begin{tabular}{lll}
\hline & Odds ratios & $95 \%$ CI \\
\hline FPS & $8.77^{* * *}$ & $(3.54 ; 21.72)$ \\
IAT & $1.06^{*}$ & $(1.01 ; 1.11)$ \\
Participants' BMI & $1.27^{*}$ & $(1.01 ; 1.60)$ \\
\hline Pseudo $R^{2}$ & 0.23 & \\
\hline
\end{tabular}

Dependent variables: contribution rates for people with obesity $(1=$ higher contributions, 0 = equal contributions); FPS, Fat Phobia Scale (higher value display more negative attitudes); BMI, body mass index; IAT, Implicit Association Test (higher value display more positive attitudes). Insignificantvariables (gender, educational attainment, household income, and age) were removed. ${ }^{*} p<0.05,{ }^{* *} p<0.01,{ }^{* * *} p \leq 0.001$.

\section{Discussion}

In this study, we aimed to investigate the public's opinion toward health insurance contributions for people with and without obesity. The analysis conducted revealed that participants suggested significantly higher health insurance contribution rates for people with obesity compared to people of normal weight. Research in other stigmatized conditions has found similar associations: Berk et al. [14], for example, conducted a representative study of the American population that assessed the public's opinion on health insurance coverage. Their study results indicate that the majority of respondents (87\%) agreed with the statement that health insurance contribution should be charged independently of a person's health status. However, more detailed questions revealed that this belief in equality was not unconditional. Participants claimed particularly an increased contribution rate for smokers $(60 \%$ of participants agreed) and for people with obesity (29\% of participants agreed). These findings among the US population might not be equally applicable for Europe and particularly for Germany due to different health care systems. To our knowledge, no comparable study assessed attitudes toward health insurance contributions of divergent risk groups in Europe.

However, the study of Lund et al. [15] examined attitudes toward public support for the treatment of obesity and lung cancer among the Danish public. In contrast to the study of Berk et al. [14], they found less support for public-funded obesity treatment compared to pulmonary diseases among (non-)smokers. Moreover, beliefs about individual failure and responsibility were found to predict less support for obesity treatment. The unequal support or rather perception of smokers and people with obesity in different countries seems noteworthy and should thus be investigated in detail. Therefore, future research should collect data in different countries, such as Germany, to examine the public's opinion on whether people of a diverse health behavior or chronic conditions that elicit different etiological beliefs, such as smokers, addicts, (high) professional athletes, or people with (type 2) diabetes should pay a higher contribution rate. That comparison of demanded health care contribution rates of divergent risk groups might give us a better understanding of where and how to rank obesity in the public eye.

Further, the multivariate analysis revealed an increased disparity of contribution rates among people with stronger explicit (FPS) and implicit stigma (IAT). These results indicate support for the assumption that people with stronger explicit and implicit attitudes toward people with obesity suggest higher contribution rates for people with obesity. However, the role of implicit antifat attitudes should be viewed with caution since we found no significant correlation between IAT and the outcome variable.

Furthermore, we found that participants with higher BMI within a (self-perceived) normal weight sample suggested higher contribution rates for people with obesity. Future 
research should consider investigating the link between BMI and attitudes toward health insurance contribution in detail.

Moreover, it seems noteworthy that overall only 38\% of participants rated the health care insurance $( \pm 10 \%)$ at the current contribution rate of $14.6 \%$. We must thus consider that the majority of participants do not know the approximate health insurance rate and therefore suggested impractical contribution rates (e.g., 100\% of employees' salary). The implemented introduction about the health care contribution system in Germany might thus not have given enough information.

The call for higher insurance rates for people with overweight or obesity can be hampered when considering a recent publication. The perception of people with obesity costing more in the health system may be short-sighted: Singh et al. [16] advocate particularly for considering weight bias as an additional cost factor. The calculation of obesity-related cost in Germany listed at the beginning of this study might thus be inchoate. Therefore, the consideration of additional costs caused by obesity and the associated weight bias might strengthen the awareness of the importance for intervention to improve the overall well-being of people affected and to reduce stigma and weight bias.

Moreover, it can be discussed whether including the premature death of people with obesity can be considered as an indirect obesity-related cost. van Baal et al., for instance, reported that "although obese people induce high medical costs during their lives, their lifetime health-care costs are lower than those of healthy-living people [...]" [17].

\section{Limitations of the Study}

The sampling procedure might limit this study. First, only participants who perceived themselves to be of normal weight were asked to contact the study team. Second, participants had to show some initiative in the form of approaching the study team via e-mail or telephone to partake in the study. Third, we recruited the majority of participants through online platforms that announce job advertisements primarily. Therefore, there is a risk for selection bias based on (a) subjective perception of body size, (b) motivation to contact the study team, and (c) recruitment through online platforms and in particular of people who searched for (parttime) job offers. Consequently, the sample cannot be described as a representative sample, in particular concerning age, educational attainment, and income. However, since this study was conducted as a pilot study, the convenience sample recruited can be justified.

Furthermore, this study was limited by participants who had a lack of knowledge regarding the statutory health care system in Germany and who therefore suggested impractical contribution rates. Some participants stated that the contribution rates should be at $100 \%$, whereas others suggested $0 \%$. However, since we did not assess the fundamental political views of participants, it remains unclear whether these impractical responses reflect political conviction or a lack of knowledge in this specific field. Future studies should therefore consider assessing covariates, such as political views or using filter questions that would explicitly detect those who do not have enough knowledge to suggest realistic contribution rates. In these cases, an adjusted question could be asked, e.g., whether people with obesity should pay a higher, lower, or equal amount as people of normal weight. Alternatively, the current (average) health care contribution rate could be stated within the short text that was implemented as a brief introduction to the topic.

\section{Statement of Ethics}

The subjects gave their written informed consent. The ethics committee of the University of Leipzig approved the study protocol (Vote No. 144-15-20042015). 
Bernard et al.: Weigh More, Pay More?

\section{Disclosure Statement}

All authors declare no conflict of interest.

\section{Funding Sources}

This study was supported by the Federal Ministry of Education and Research (BMBF), Germany (FKZ: 01E01501). The funding source was not involved in the study design, collection, analysis, and interpretation of data. Furthermore, we acknowledge support from the German Research Foundation (DFG) and the University of Leipzig within the program of Open Access Publishing.

\section{Author Contributions}

M.B., C.L.-S., and S.G.R.-H. outlined and specified the research question. M.B. and C.L.-S. conducted the statistical analysis. M.B. wrote the first draft of the manuscript. S.G.R.-H. and C.L.-S. revised the manuscript critically for relevant intellectual content. All authors contributed to and approved the final version of the manuscript.

\section{References}

1 Effertz T, Engel S, Verheyen F, Linder R. The costs and consequences of obesity in Germany: a new approach from a prevalence and life-cycle perspective. Eur J Health Econ. 2016 Dec;17(9):1141-58.

2 Klein S. Weißbuch Adipositas: Versorgungssituation in Deutschland Gutachten für Johnson \& Johnson Medical GmbH. Berlin: Medizinisch Wissenschaftliche Verlagsgesellschaft; 2016.

3 Schlegel R. German health care-towards universal access. An overview of the principles of access and benefits. J Contemp Health Law Policy. 2002;18(3):673-83.

4 Bundesministerium für Gesundheit. Beiträge und Tarife, 2018 [cited 2018 November 6]. Available from: https:// www.bundesgesundheitsministerium.de/beitraege-und-tarife.html.

5 Bundesversicherungsamt: So funktioniert der neue Risikostrukturausgleich im Gesundheitsfonds 2008. Available from: https://www.bundesversicherungsamt.de/fileadmin/redaktion/Risikostrukturausgleich/Wie_ funktioniert_Morbi_RSA.pdf.

6 Bundesversicherungsamt: Übersicht über die für das Ausgleichsjahr 2016 zu berücksichtigenden Krankheiten nach § 31 Abs. 2 RSAV 2015. Available from: https://www.bundesversicherungsamt.de/fileadmin/redaktion/ Risikostrukturausgleich/01a_Krankheitsauswahl_AJ2016_nur_Krankheiten_.pdf.

7 Andreyeva T, Puhl RM, Brownell KD. Changes in perceived weight discrimination among Americans, 1995-1996 through 2004-2006. Obesity (Silver Spring). 2008 May;16(5):1129-34.

8 Puhl RM, Heuer CA. The stigma of obesity: a review and update. Obesity (Silver Spring). 2009 May;17(5):941-64.

9 Woolford SJ, Clark SJ, Butchart A, Geiger JD, Davis MM, Fagerlin A. To pay or not to pay: public perception regarding insurance coverage of obesity treatment. Obesity (Silver Spring). 2013 Dec;21(12):E709-14.

10 Greenwald AG, McGhee DE, Schwartz JL. Measuring individual differences in implicit cognition: the implicit association test. J Pers Soc Psychol. 1998 Jun;74(6):1464-80.

11 Bacon JG, Scheltema KE, Robinson BE. Fat phobia scale revisited: the short form. Int J Obes Relat Metab Disord. 2001 Feb;25(2):252-7.

12 StataCorp. Stata Statistical Software. TX: StataCorp LP; 2015.

13 Kohler U, Kreuter F. Datenanalyse mit Stata: Allgemeine Konzepte der Datenanalyse und ihre praktische Anwendung. 5., aktualisierte Auflage. Berlin, Boston: De Gruyter Oldenbourg; 2017.

14 Berk ML, Gaylin DS, Schur CL. Exploring the public's views on the health care system: a national survey on the issues and options. Health Aff (Millwood). 2006 Nov-Dec;25(6 Suppl1):w596-606. Available from: https://www. healthaffairs.org/doi/pdf/10.1377/hlthaff.25.w596

15 Lund TB, Nielsen ME, Sandøe P. In a class of their own: the Danish public considers obesity less deserving of treatment compared with smoking-related diseases. Eur J Clin Nutr. 2015 Apr;69(4):514-8.

16 Singh K, Russell-Mayhew S, von Ranson K, McLaren L. Is there more to the equation? Weight bias and the costs of obesity. Can J Public Health. 2019 Feb;110(1):17-20.

17 van Baal PH, Polder JJ, de Wit GA, Hoogenveen RT, Feenstra TL, Boshuizen HC, et al. Lifetime medical costs of obesity: prevention no cure for increasing health expenditure. PLoS Med. 2008 Feb;5(2):e29. 